\title{
Portion size and energy contribution of food in adolescents from São Paulo, Brazil: Changes between 2003 and 2015
}

\section{Abstract}

Introduction: Overweight has become a very important issue in the epidemiological scenario of nutrition due to its increasing rates and association with several chronic noncommunicable diseases. The etiology of overweight is complex, but diet is one of the modifiable factors that may interfere in the nutritional status of individuals and consequently on overweight and obesity. One aspect of the diet that has been related to overweight is the increase of energy intake, and related to that, the increase on food portion size.

Objective: To evaluate differences in food portions size and its energy contribution in adolescents from São Paulo, Brazil between 2003, 2008 and 2015.

Materials and Methods: Cross-sectional population-based study with a representative sample of 1711 adolescents aged 12 to 19 years who participated in the Health Survey of São Paulo (HS-SP). The HS-SP was conducted in 2003, 2008 and 2015. Overweight was defined according to the cutoff points of the World Health Organization (WHO) for adolescents (BMI $\geq$ p85). Dietary data were obtained from two 24-hour recalls.Reported foods were classified into groups and the prevalence of consumers, the percentage of energy contribution, and the mean and median of portion sizes in each food group were calculated.

Results: Over the years, the prevalence of overweight in adolescents increased from $13.8 \%$ to $29.4 \%$. The prevalence of consumers of salty snacks, fruits, industrialized juices, red meats, rice, sweets/desserts and toast/cookies has increased; while the number of consumers of coffee/tea, fruit juice, milk, artisan/non-packaged snacks and sugar decreased. The food groups with the highest energy contribution were: rice $(14 \%)$, red meat $(12 \%)$ and bread $(9 \%)$ in all the years evaluated. The groups with the greatest increase in the energy contribution from 2003 to 2015 were: toast / cookies, sweets / desserts, white and red meats, and industrialized juices. The consumption portion size of eggs has increased, while those of cold cuts, fruits, industrialized juices, rice and tubers/roots decreased.

Discussion: There was a diverse variation in the frequency of intake, the energy contribution and the food portion size over time with increase in some groups and decrease in others. These findings provide useful data to support future interventions and public policies focused on adolescent feeding.

\section{Conflict of Interest}

There is no conflict of interest. 